\title{
Long-term outcome of myocardial revascularization in patients with Kawasaki coronary artery disease
}

\author{
A multicenter cooperative study
}

The long-term outcome of myocardial revascularization by coronary artery bypass grafting in patients with severe coronary obstruction caused by Kawasaki disease is largely unknown. A multicenter follow-up study was performed in 1991. A total of 168 patients with Kawasaki disease (127 male [75.6\%] and 41 female patients [ $24.4 \%$ ]) who had undergone coronary bypass grafting were enrolled. Obstructive coronary artery disease affected the left main trunk in $11.8 \%$, the right coronary artery in $\mathbf{7 7 . 6 \%}$, the left anterior descending in $87.6 \%$, and the left circumflex in $25.9 \%$. Old myocardial infarction was noted in $46.0 \%$ of the patients. Fifty-four patients (32\%, $12.4 \pm 9.8$ years) underwent bypass grafting with saphenous vein grafts alone. The remaining 114 patients $(68 \%, 9.8 \pm 7.1$ years) received at least one internal thoracic artery graft to the left anterior descending coronary artery. Gastroepiploic artery grafts were used in 12 patients. There were no significant differences between the saphenous vein and internal thoracic artery groups in the mean age at operation (12.4 versus 9.8 years), female ratio (22\% versus $25 \%$ ), the number of patients over 20 years of age $(9.3 \%$ versus $9.6 \%$ ), previous history of infarction $(51.9 \%$ versus $41.2 \%$ ), impaired left ventricular function (ejection fraction $<0.5)(13.0$ versus $11.4 \%)$, left main trunk disease $(11.1 \%$ versus $10.5 \%)$, the number of vessels involved $(2.2 \pm 0.8$ versus $2.0 \pm 0.6$ per patient $)$, or the mean number of grafts used $(1.7 \pm 0.7$ versus $1.7 \pm 0.7$ per patient). The operative death rate was also the same in the two groups $(1.9 \%$ versus $0 \%$ ), but the late cardiac death rate was significantly higher in the saphenous vein graft group $(13.0 \%)$ than in the internal thoracic artery group $(0.9 \%)(p<0.003)$. Actuarial analysis showed a significantly higher survival in the internal thoracic artery group $(98.7 \% \pm 1.2 \%$ versus $81.6 \% \pm 7.0 \%, p<0.05)$ at 90 months after the operation. Late death was strongly related to the absence of an internal thoracic artery graft $(p<0.003)$ and to the age at the time of operation $(p<0.05)$. The actuarial patency rate was significantly higher for arterial grafts $(77.1 \% \pm 1.1 \%$, $n=151)$ than for vein grafts $(46.2 \% \pm 6.3 \%, n=126) 85$ months after the operation $(p<0.003)$. Arterial grafts were used for the non-left anterior descending coronary arteries in only 41 of 155 grafts $(26.5 \%)$; in contrast, vein grafts were used in 85 of 133 grafts $(63.9 \%)(p<0.005$ to 0.001$)$. However, the actuarial patency rate was significantly higher for arterial grafts $(81.4 \% \pm 8.2 \%$,

Soichiro Kitamura, $\mathrm{MD}^{\mathrm{a}}$ (by invitation), Yoichi Kameda, $\mathrm{MD}^{\mathrm{a}}$ (by invitation), Toshio Seki, MD (by invitation), Kanji Kawachi, MDa (by invitation), Nara, Japan, Masahiro Endo, $\mathrm{MD}^{\mathrm{b}}$ (by invitation), Yasuo Takeuchi, $\mathrm{MD}^{\mathrm{c}}$ (by invitation), Tomisaku Kawasaki, MD ${ }^{\mathrm{d}}$ (by invitation), Tokyo, Japan, Yasunaru Kawashima, MD, Osaka, Japan, and the investigators of the Multicenter Cooperative Study

From the Department of Surgery III, Nara Medical College, Nara ${ }^{a}$; the Department of Cardiovascular Surgery, Tokyo Women's Medical College, Tokyo ${ }^{b}$; the Department of Cardiovascular Surgery, Kanto Teishin Hospital, Tokyo ${ }^{\mathrm{c}}$; the Information Center for Kawasaki Disease, Tokyo ${ }^{d}$; and the National Cardiovascular Center, Osaka, Japan. $^{e}$

Supported by a grand-in-aid from the Japanese Ministry of Health and Welfare.
Read at the Seventy-third Annual Meeting of The American Association for Thoracic Surgery, Chicago, Ill., April 25-28, 1993.

Address for reprints: Soichiro Kitamura, MD, Department of Surgery III, Nara Medical College, 840 Shijo, Kashihara, Nara 634, Japan.

J THORAC CARDIOVASC SURG 1994;107:663-74

Copyright $\odot 1994$ by Mosby-Year Book, Inc.

$0022-5223 / 94 \$ 3.00+0 \quad 12 / 6 / 51775$ 
$n=39)$ than for vein grafts $(37.5 \% \pm 8.0 \%, n=81)$ to the left circumflex and right coronary arteries. The difference in patency was even greater in children younger than 7 years old at operation $(p<0.005)$. In conclusion, internal thoracic artery grafts showed significantly better long-term patency than saphenous vein grafts in patients with Kawasaki disease, and the use of internal thoracic artery grafts reduced the likelihood of late cardiac death. (J THORAC CARDIOvASC SURG 1994;107:663-74)

\begin{abstract}
A present, the leading cause of pediatric ischemic heart disease is Kawasaki disease in many countries around the world. Coronary artery obstructive disease of inflammatory origin can occur any time from 1 to 20 years after the acute illness, and it develops at the site of aneurysm formation in most patients. Because the lesions are commonly near the origin of the left and right coronary arteries, the feasibility and efficacy of surgery had been advocated. ${ }^{1}$ In 1974, coronary artery bypass grafting with autologous saphenous vein grafts (SVGs) was̀ conducted in children and adolescents for the first time. ${ }^{1,2}$ Since then, myocardial revascularization for coronary lesions resulting from Kawasaki disease has been conducted increasingly in Japan, ${ }^{3-5}$ and reports of such operations are also recently increasing in the United States. ${ }^{6-9}$ Subsequent follow-up revealed that SVGs frequently become obstructed within 1 to 2 years. ${ }^{3-5}$ Accordingly, the use of internal thoracic artery (ITA) grafts was first attempted in $1983,{ }^{10}$ followed by the successful use of both gastroepiploic artery (GEA) and ITA grafts. ${ }^{11-14}$

Since the initial surgical treatment for coronary artery lesions caused by Kawasaki disease was performed, more than 10 years have passed. However, the long-term surgical outcome in a large series of patients is unknown. Therefore, a multicenter cooperative study was undertaken in 1991 under the auspices of the Japanese Ministry of Health and Welfare. This report provides data on the outcome of myocardial revascularization in 170 patients with Kawasaki coronary artery disease. Particular emphasis is placed on comparison of the differences in the late results in patients receiving arterial grafts or venous grafts for myocardial revascularization as children.
\end{abstract}

\section{Patients and methods}

In 1991, a multicenter group study was undertaken by a mailed questionnaire to collect postoperative data on the patients who had undergone myocardial revascularization for coronary artery lesions developing as a result of Kawasaki coronary arteritis (mucocutaneous lymph node syndrome). ${ }^{15} \mathrm{~A}$ total of 170 patients with a mean age of $10.6 \pm 8.1$ years at the time of operation were enrolled from the 40 institutions listed in the appendix of this report. There were 129 male $(75.6 \%)$ and 41 female patients $(24.4 \%$ ), for a male/female ratio of approximately 3:1. The age and sex distributions of the patients are shown in Fig. 1. The ages ranged from 1 to 44 years, with a peak around 5 to 7 years. There were 16 adults $(9.4 \%)$ aged over 20 years who were also enrolled without exclusion in this study because of their apparent histories, characteristic angiographic findings, or the complete lack of risk factors for atherosclerosis. The actual surgical strategy was not different from that used in the younger patients. The mean ages of the male and female patients were $10.3 \pm 7.7$ and $11.6 \pm 9.4$ years $(p=$ not significant), respectively.

Coronary artery aneurysms were identified in 161 patients $(94.7 \%)$ by selective coronary arteriography. The aneurysm was located at the left main trunk in $45.3 \%$ of the patients, at the right coronary artery (RCA) in $70.0 \%$, at the left anterior descending artery (LAD) in $53.5 \%$, and at the left circumflex artery in $24.1 \%$. Obstructive coronary artery disease that had developed as a consequence of aneurysm formation was identified before operation at the following sites: left main trunk in $11.8 \%$ of the patients, RCA in $77.6 \%$, LAD in $87.6 \%$, and left circumflex in $25.8 \%$ (Fig. 2). Obstructive disease almost always developed at the inflow or outflow site of the coronary aneurysm. Aneurysms of the left main trunk eventually produced obstructive lesions of the LAD in about one third of the patients. As a result, the LAD was the most common artery with obstruction, followed by the RCA, left circumflex, and left main trunk in that order. Lesions of the left main trunk were seen in $11.8 \%$ of the patients before the operation. Among the 170 patients enrolled in this study, only two had coronary aneurysms alone without clear stenotic lesions. The remaining 168 patients had angiographic obstruction and their lesions were classified as singlevessel disease $(18.5 \%)$, double-vessel disease $(48.8 \%)$, triplevessel disease $(20.8 \%)$, and left main trunk disease (11.9\%) (Table I). Among the 163 patients with data available, 75 $(46.0 \%)$ had had a myocardial infarction at least as judged by electrocardiography. As shown in Table II, the most common site of myocardial infarction was the inferior wall (27.0\%), followed by anteroseptal infarction $(20.2 \%)$, lateral infarction $(11.7 \%)$, posterior infarction $(2.5 \%)$, or right ventricular infarction $(1.8 \%)$ in that order. Twenty-eight patients had infarcts at more than one location. The left ventricular ejection fraction ranged from 0.27 to 0.77 , with a mean of $0.56 \pm 0.13$, but this information was available in only 79 patients $(46.5 \%)$.

Coronary artery bypass grafting was performed in 168 patients $(98.8 \%)$ and left main trunk repair was done in two patients $(1.2 \%)$. Among the 168 patients undergoing coronary bypass, $72(42.3 \%)$ received a single graft (usually to the LAD), $74(43.5 \%)$ received two grafts, $20(11.8 \%)$ received three grafts, and two $(1.2 \%)$ received four grafts. Thus a total of 288 grafts were used in 168 patients. The mean number of grafts per patient was $1.7 \pm 0.7$. Fifty-four of the 168 patients $(32.1 \%)$ underwent coronary bypass with autologous SVGs alone (SVG group) and the remaining 114 patients $(67.9 \%)$ had at least one ITA graft to the LAD (ITA group). GEA grafts were used in 


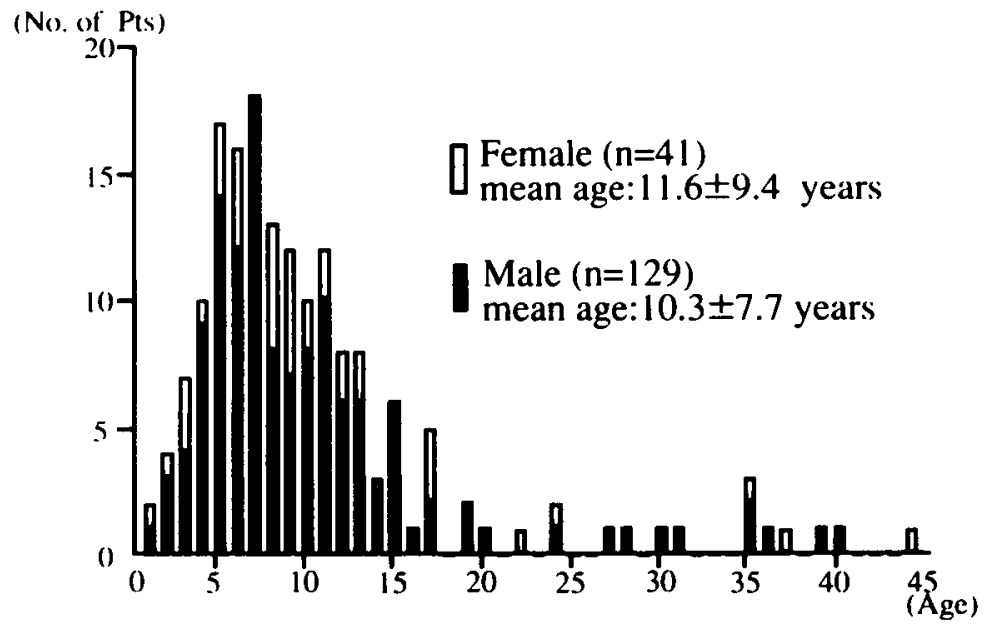

Fig. 1. Age distribution of the 170 male and female patients at the time of operation. The mean age was $10.6 \pm 8.1$ years, ranging from 1 to 44 years with a peak at 5 to 7 years. About one quarter of the patients were female. Sixteen adults $(9.4 \%)$ over 20 years of age were also enrolled in this multicenter study because of their characteristic angiographic findings and the complete lack of risk factors for atherosclerotic heart disease.

Table I. Number of vessels with obstructive lesions

\begin{tabular}{lcc}
\hline & $\begin{array}{c}\text { No. of } \\
\text { patients }\end{array}$ & Percent \\
\hline SVD & 31 & 18.5 \\
DVD & 82 & 48.8 \\
TVD & 35 & 20.8 \\
Isolated LMT & 5 & 2.9 \\
+SVD & 8 & 4.811 .9 \\
+DVD & 4 & 2.4 \\
+TVD & 3 & 1.8 \\
Total No. of patients & $\underline{168}$ & \\
\hline
\end{tabular}

Two patients had coronary aneurysms alone and are not shown in this table. $S V D$ Single vessel disease, $D V D$, double vessel disease; $T V D$, triple vessel disease, $L M T$, left main trunk lesion

Table II. Previous myocardial infarction $75 / 163$, or $46.0 \%$ )

\begin{tabular}{lcc}
\hline \multicolumn{1}{c}{ Location } & No. & Percent \\
\hline Anteroseptal & 33 & 20.2 \\
Lateral & 19 & 11.7 \\
Inferior & 44 & 27.0 \\
Posterior & 4 & 2.5 \\
Right ventricular & 3 & 1.8 \\
\hline
\end{tabular}

Twenty-eight patients had infarcts at more than one location.

12 patients. As shown in Table III, there were no differences between these two groups with respect to the mean age, sex ratio, the number of patients over 20 years of age, prevalence of myocardial infarction, the number of vessels involved, prevalence of left main trunk disease, prevalence of a left ventricular ejection fraction less than 0.5 , and the number of grafts. Left main trunk lesions were counted as double-vessel disease when the number of vessels involved was being calculated.

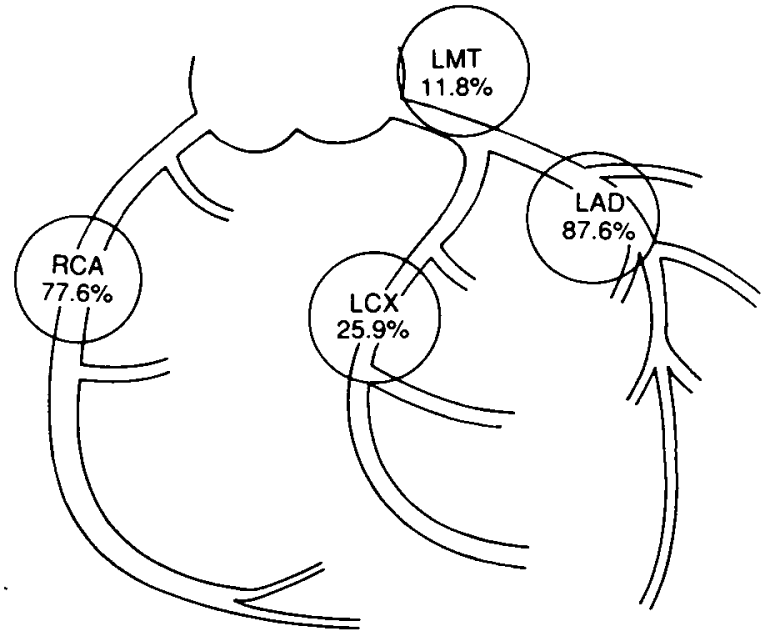

Fig. 2. Coronary artery obstruction caused by Kawasaki disease in surgical patients. Obstructive disease developed at the inflow or outflow sites of the coronary aneurysms, which were generally located in the proximal part of the coronary artery tree. The left anterior descending artery $(L A D)$ was most commonly involved, followed by the right coronary artery $(R C A)$, left circumflex artery $(L C X)$, and the left main trunk $(L M T)$ in that order.

Comparisons of target coronary arteries and severity of obstruction for arterial and venous bypass grafts are shown in Table IV. The severity of the stenosis in the native coronary arteries was assessed only as totally or partially obstructed in this study. Among the 288 grafts used, 155 were arterial (143 ITAs, 12 GEAs) and 133 were venous. Among these, 51 (32.9\%) arterial and $40(30.1 \%)$ venous grafts were anastomosed to totally obstructed coronary arteries, and 104 (67.1\%) arterial 


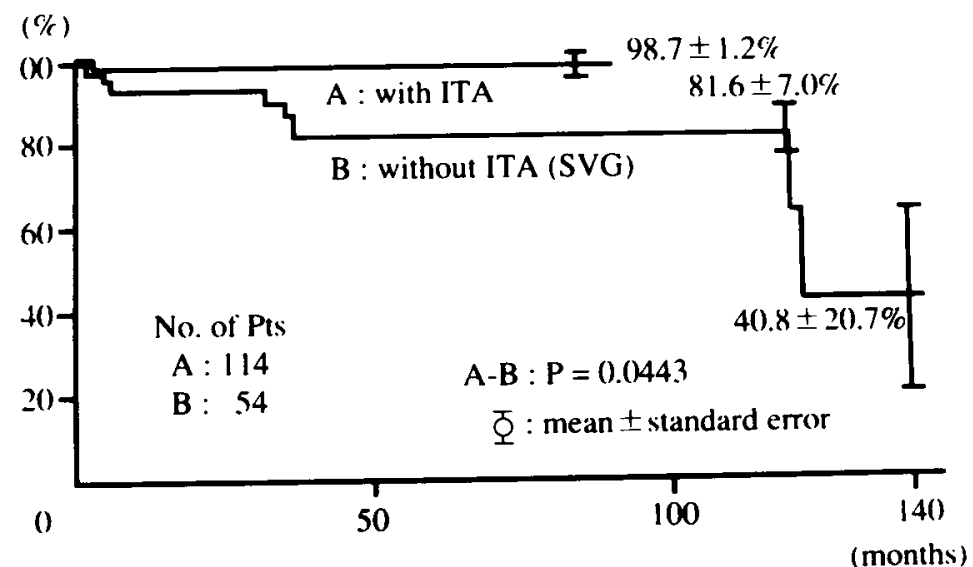

Fig. 3. Actuarial survival of patients with at least one ITA to the LAD coronary artery (ITA group) and patients with only SVGs (SVG group). There was a significant difference in survival at 90 months after the operation $(p<0.05)$. The use of an ITA graft was a strong $(p<0.003)$ predictor of better late postoperative survival.

Table III. Demographic data of the SVG group and the ITA group

\begin{tabular}{lccc}
\hline & SVG group & ITA group & Statistical significance \\
\hline No. of patients & 54 & 114 & NS \\
Age (yr) & $12.4 \pm 9.8$ & $9.8 \pm 7.1$ & NS \\
No. of patients $>20$ yr of age & $9.3 \%$ & $9.6 \%$ & NS \\
Gender (M/F) & $42 / 12(77.8 \%)$ & $85 / 29(74.6 \%)$ & NS \\
History of MI & $51.9 \%$ & $41.2 \%$ & NS \\
Anterior MI & $27.8 \%$ & $15.9 \%$ & NS \\
Impaired LV function (EF $<0.5)$ & $13.0 \%$ & $11.4 \%$ & NS \\
LMTD & $11.1 \%$ & $10.5 \%$ & NS \\
No. of vessels involved & $2.2 \pm 0.8 / \mathrm{pt}$ & $2.0 \pm 0.6 / \mathrm{pt}$ & NS \\
No. of grafts & $1.7 \pm 0.7 / \mathrm{pt}$ & $1.7 \pm 0.7 / \mathrm{pt}$ & \\
ITA graft to LAD & $0 \%$ & $100 \%$ & NS \\
Operative death & $1.9 \%$ & $0 \%$ & $p<0.003$ \\
Late death & $13.0 \%$ & $0.9 \%$ & \\
\hline
\end{tabular}

SVG Saphenous vein graft: $I T A$, internal thoracic artery; $M I$, myocardial infarction; $L V$, left ventricular; $E F$, ejection fraction; $L M T D$, left main trunk disease; $S V G$, Saphenous vein gresel disease. Mean values are shown as the mean \pm SD. Differences in mean values were assessed by the Wilcoxon test and differences in percentages were determined by the $\chi^{2}$ test with Yates' correction.

and $93(69.9 \%)$ venous grafts were anastomosed to partially obstructed (stenotic) coronary arteries. There were no significant differences between arterial and venous grafts in regard to the severity of obstruction of the target vessel. For the LAD, 114 (73.5\%) arterial and $48(36.1 \%)$ venous grafts were used; for the left circumflex artery, $6(3.9 \%)$ arterial and $34(25.6 \%)$ venous grafts; and for the RCA, $35(22.6 \%)$ arterial and $51(38.3 \%)$ venous grafts were anastomosed. Vein grafts were used for nonLAD arteries (left circumflex and RCA) 2.5 times more frequently than arterial grafts $(p<0.005$ to 0.001$)$. For this reason, patency rates between arterial and venous grafts were compared for coronary arteries as a whole as well as for the LAD and non-LAD arteries. Postoperative angiographic follow-up was performed for $151(97.4 \%)$ arterial and $126(94.7 \%)$ venous grafts; the rate of angiographic follow-up was similar for the two types of grafts.

Surgical techniques and the methods of myocardial protec- tion during the operations were different at each institution. However, no major differences in surgical and adjunctive techniques existed for arterial and venous bypass graft procedures. All patients had at least antegrade (aortic) cold cardioplegic infusion with either crystalloid or blood solution during aortic crossclamping. Because there was no difference in the early mortality between the ITA and SVG groups (Table III), surgical adjunctive techniques appeared to have little effect on other comparisons.

Data analysis. For statistical assessment, unpaired Student's $t$ test or the Wilcoxon rank test was used for examining the differences in mean values between the two groups, and the $\chi^{2}$ test with Yates' correction was used for examining percentages. Graft patency and patient survival were analyzed by the Kaplan-Meier method and differences were assessed by the generalized Wilcoxon test or the log rank test. The mean percentage values obtained by actuarial analysis are shown as the 
Table IV. Target coronary arteries and the severity of obstruction

\begin{tabular}{|c|c|c|c|c|c|}
\hline & \multicolumn{2}{|c|}{ Arterial grafts } & \multicolumn{2}{|c|}{ Venous grafts } & \multirow{2}{*}{$\begin{array}{l}\text { Statistical } \\
\text { significance }\end{array}$} \\
\hline & No. & $\%$ & No. & $\%$ & \\
\hline Total numbers used & 155 & & 133 & & \\
\hline ITA & 143 & & & & \\
\hline GEA & 12 & & & & \\
\hline \multicolumn{6}{|l|}{ Target coronary arteries } \\
\hline LAD & 114 & 73.5 & 48 & 36.1 & $p<0.001$ \\
\hline $\mathrm{LCX}$ & 6 & 3.9 & 34 & 25.6 & $p<0.001$ \\
\hline $\mathrm{RCA}$ & 35 & 22.6 & 51 & 38.3 & $p<0.005$ \\
\hline \multicolumn{6}{|l|}{ Severity of target coronary artery obstruction } \\
\hline Total occlusion & 51 & 32.9 & 40 & 30.1 & NS \\
\hline Partial stenosis & 104 & 67.1 & 93 & 69.9 & NS \\
\hline Postop. angiographic follow-up available & 151 & 97.4 & 126 & 94.7 & NS \\
\hline
\end{tabular}

$L A D$, Left anterior descending artery; $L C X$, left circumflex artery; $R C A$, right coronary artery.

Table V. Causes of death

\begin{tabular}{cccccc}
\hline & & & \multicolumn{2}{c}{ Causes } \\
\cline { 4 - 6 } & No. & Age $(y r)$ & Sex $(M, F)$ & Mode & No. \\
\hline Early death & 2 & 7,8 & 1,1 & AMI & LOS \\
Late death & $(1.2 \%)$ & & & Sudden & 1 \\
& $(4.7 \%)$ & $5.9 \pm 4.3$ & & AMI & 5 \\
& & & CHF & 2 & 1
\end{tabular}

AMI, Acute myocardial infarction; $L O S$, low cardiac output syndrome; $C H F$, congestive heart failure. One late death in the ITA group was sudden; the other sudden deaths were all in the SVG group.

mean \pm standard error, and other mean values are reported as the mean \pm standard deviation. Differences were considered statistically significant at a $p$ value less than 0.05 .

The multivariate logistic method is preferable to the univariate method performed for multicenter data analysis and increases the validity of data evaluation. Unfortunately, however, the unavailability of important data in many patients, such as left ventricular ejection fraction, precluded accurate multivariate analysis in this study.

\section{Results}

Survival. Two early (in-hospital) deaths and eight late deaths occurred among the 170 surgical patients during follow-up to the end of 1991. One early death occurred in the SVG group and the other hospital death occurred in one of the two patients undergoing left main trunk repair. Early death was due to perioperative acute myocardial infarction in one patient and to postoperative low cardiac output syndrome in the other. In the SVG group, one hospital death $(1.9 \%)$ and seven late deaths (13.0\%) occurred during a follow-up period of 140 months (Table III). In contrast, no hospital deaths $(0 \%)$ and only one late death $(0.9 \%)$ occurred in the ITA group during a followup period of 96 months. The late death rate of the two groups was significantly different when compared by the $\chi^{2}$ test with Yates' correction or Fisher's exact test $(p<0.003)$. Eight late deaths were of cardiac origin and occurred in patients with a mean age at operation of $5.9 \pm 4.3$ years, ranging from 1 to 14 years (Table V). The most common mode of late death was sudden death, which occurred in five patients and was confirmed to be due to ventricular fibrillation in three of them. Late death occurred at $45 \pm 49$ months (range: 2 to 122 months) after bypass grafting. The mean ejection fraction in the patients dying late in the postoperative period was $0.50 \pm 0.11$, and it was lower than that of the surviving patients $(n=73,0.57 \pm 0.13)$ but the difference was not significant. Univariate analysis demonstrated that the age at operation was a significant $(p<0.05)$ risk factor for hospital and late death. The mean age at operation in the 10 patients who died in the postoperative period was $6.2 \pm 3.9$ years (mean \pm standard deviation), and it was $10.9 \pm 8.2$ years in the 160 survivors $(p<0.05)$. Gender did not correlate with death.

The actuarial analysis of survival in the SVG and ITA groups is shown in Fig. 3. The ITA group ( $A$ in Fig. 3) had a significantly better survival of $98.7 \% \pm 1.2 \%$ (mean \pm standard error) than did the SVG group$81.6 \% \pm 7.0 \%$ at 10 years and $40.8 \% \pm 20.7 \%$ at 12 


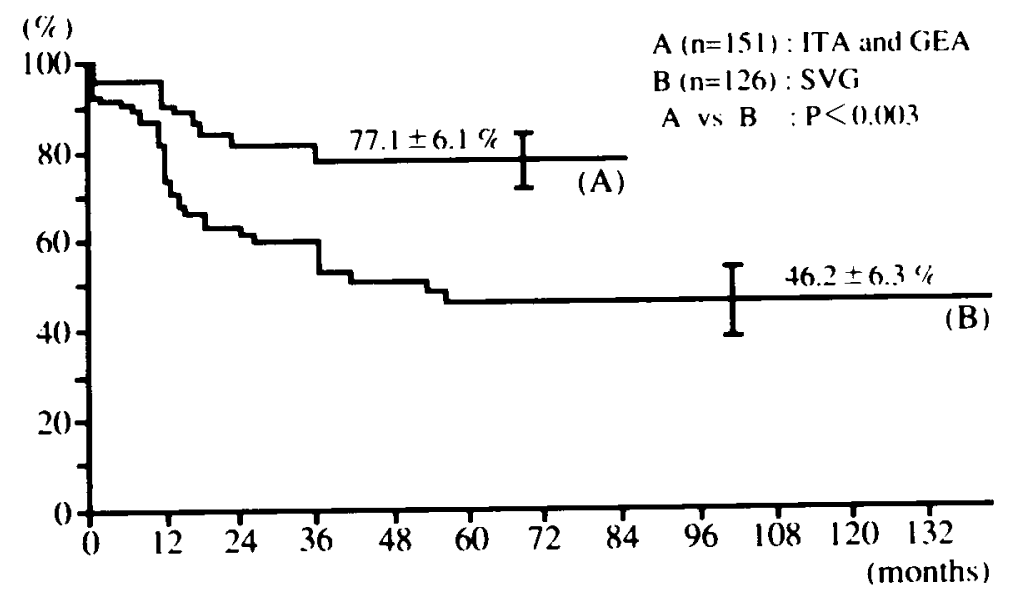

Fig. 4. Comparison of actuarial graft patency rates between arterial grafts $(A)(n=151)$ and venous grafts $(B)$ $(n=126)$. Arterial grafts showed a significantly higher angiographic patency than venous grafts. Occlusion of venous grafts was common during the first few years after operation.

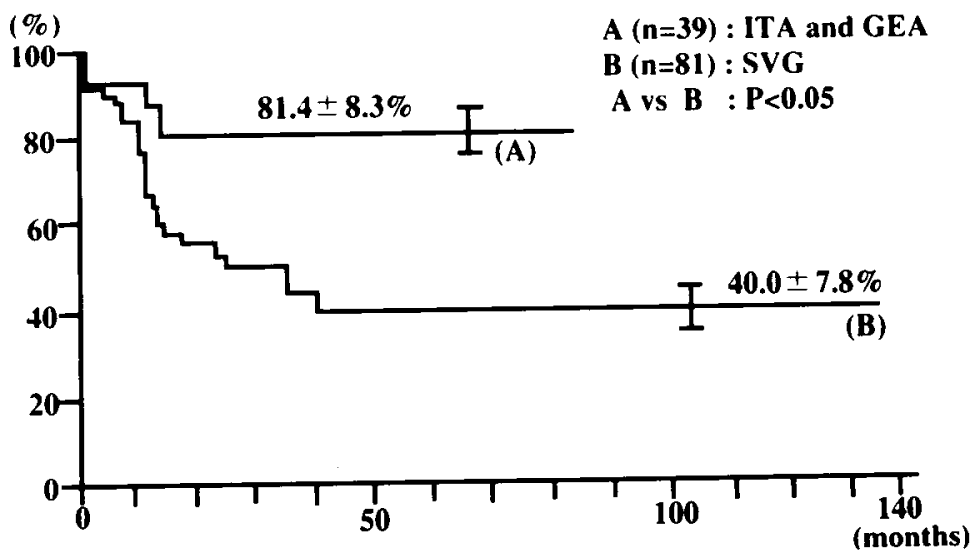

Fig. 5. Comparison of actuarial graft patency rates between arterial grafts $(A)(n=39)$ and venous grafts $(B)$ $(n=81)$ for the right and circumflex arteries. Arterial grafts also showed a significantly higher patency than venous grafts.

years ( $B$ in Fig. 3) $(p<0.05$, generalized Wilcoxon test). Univariate analysis also showed that the use of the ITA grafts was a strong $(p<0.003)$ predictor of a better late postoperative survival in these patients with severe coronary artery lesions caused by Kawasaki disease.

Graft patency. Graft patency was confirmed by postoperative angiography. Actuarial patency for 151 arterial grafts (140 ITA and 11 GEA grafts) and 126 SVGs is compared in Fig. 4. The arterial graft patency rate 85 months after the operation was $77.1 \% \pm 6.1 \%$ (mean \pm standard error), and it was $46.2 \% \pm 6.3 \%$ for SVGs $(p<0.003$, generalized Wilcoxon test). When the 11 GEA grafts were excluded from the arterial group, the patency rate for ITA grafts $(n=140)$ alone was
$77.7 \% \pm 6.2 \%$, and the difference from the SVG group was even more significant $(p<0.001)$.

Because vein grafts were used approximately 2.5 times (63.9\%) more frequently than arterial grafts $(26.5 \%)$ for the non-LAD vessels (RCA and left circumflex), graft patency was compared for arterial and venous grafts for non-LAD lesions (Fig. 5). Here again, the arterial graft $(n=39,81.4 \% \pm 8.3 \%)$ showed a significantly higher patency rate than venous grafts $(n=81,40.0 \% \pm 7.8 \%)$ for the RCA and left circumflex $(p<0.05)$ at the eightyfifth postoperative month. However, a comparison of patency rates for arterial and venous grafts for the LAD did not reach statistical significance $(p=0.236)$, although the patency rate for arterial grafts was superior 


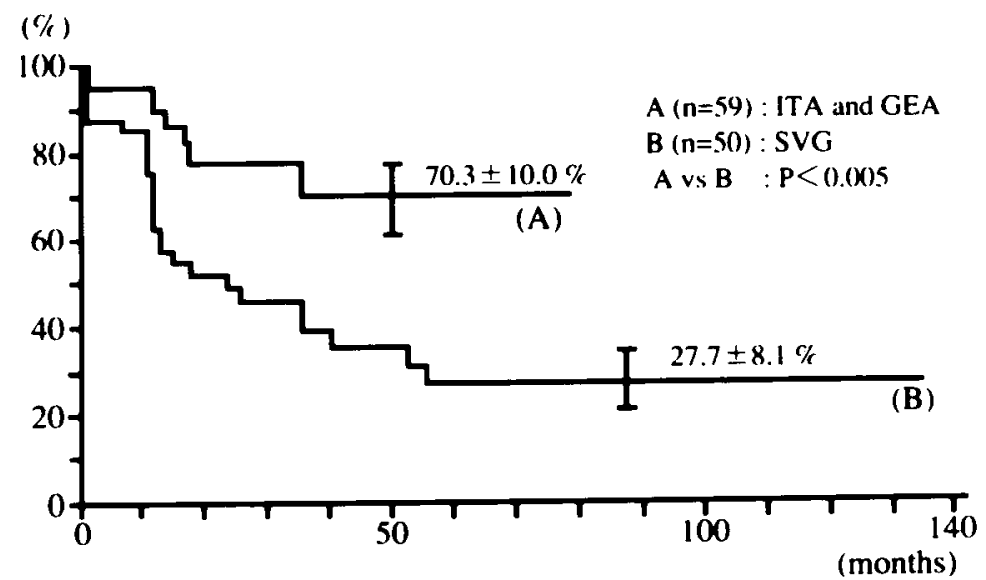

Fig. 6. Comparison of SVG and arterial graft patency in patients under 7 years of age at operation. SVG patency was only $27.7 \% \pm 8.1 \%$ and arterial graft patency was $70.3 \% \pm 10.0 \%$ at 84 months after the operation $(p<0.005)$.

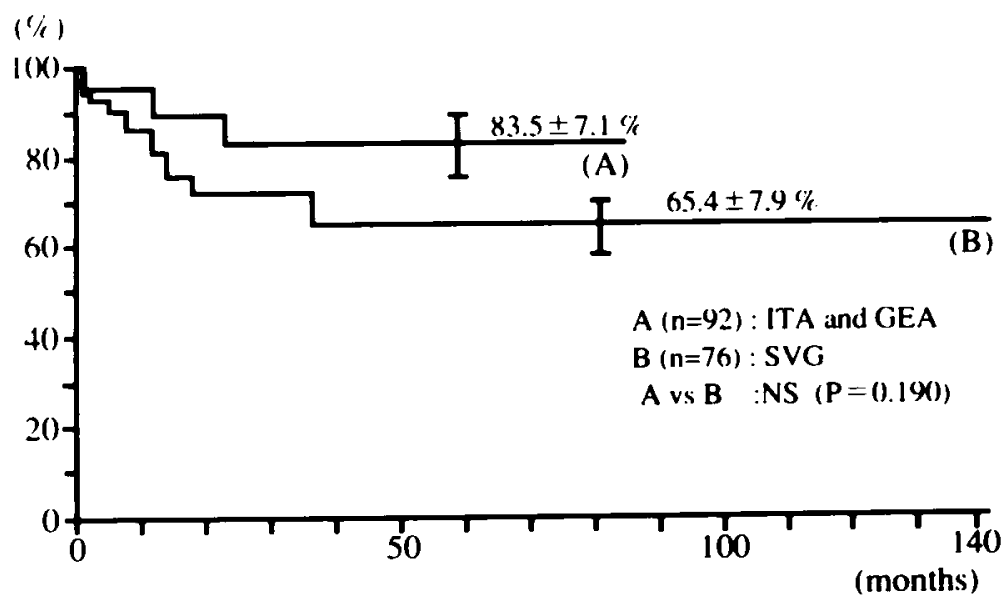

Fig. 7. Comparison of SVG and arterial graft patency in patients over 8 years of age. SVG patency was $65.4 \% \pm 7.9 \%$ and arterial graft patency was $83.5 \% \pm 7.1 \%$ at 90 months after the operation ( $p=$ not significant).

to that of venous grafts $(75.8 \% \pm 7.1 \%$ versus $57.2 \% \pm 10.1 \%$ ) at the same follow-up periods.

Patency of SVGs was particularly poor in small children. The cutoff age for a decrease of SVG patency was 7 years. The patency rate was compared between 59 arterial grafts and $50 \mathrm{SVGs}$ in patients under 7 years of age (Fig. 6). The patency rate for SVGs was particularly poor $(27.7 \% \pm 8.1 \%)$ in this age group, and that of arterial grafts was $70.3 \% \pm 10.0 \%(p<0.005)$. In contrast, SVG patency was much better in patients over 8 years of age $(65.4 \% \pm 7.9 \%, n=76)$, and the patency rate for arterial grafts was also better $(83.5 \% \pm 7.1 \%)$ (Fig. 7). However, there was no significant difference $(p=0.190)$ in the patency of arterial and venous grafts in this age group after follow-up for 85 and 140 months, respectively.

When the 16 adults were excluded, the patency for the arterial grafts $(n=143)$ was $77.4 \% \pm 6.2 \%$ at the eighty-fifth postoperative month and that of venous grafts $(n=105)$ was $44.5 \% \pm 6.4 \%(p<0.001)$. These results are similar to those for the entire group. Inclusion or exclusion of these adult patients did not appreciably affect any important results, such as graft patency or survival rates.

Postoperative clinical status and cardiac events other than early death. The postoperative clinical status was analyzed in 168 patients, excluding the two with early death. Eighty-four percent of the patients were in good 


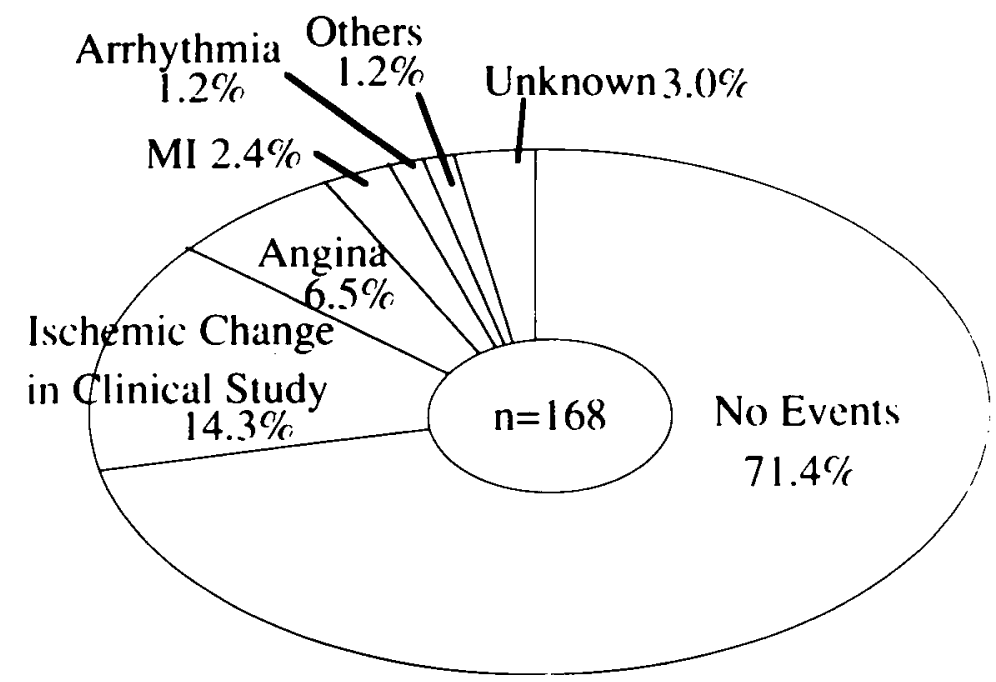

Fig. 8. Postoperative cardiac events. Approximately $70 \%$ of the patients had no cardiac events after the operation. There was a relatively high overall postoperative event rate of $24.4 \%$, but there were no differences in the prevalence of nonfatal cardiac events between the patients with ITA grafts $(24.6 \%)$ and those with only SVGs $(24.1 \%) . M I$, Myocardial infarction.

health, $12.5 \%$ showed no improvement, $3.0 \%$ had worsened, and the outcome was unknown for $0.6 \%$. Postoperative cardiac events reported in the 168 patients who survived the operation are shown in Fig. 8. There were no postoperative cardiac events in $71.4 \%$ of the patients. Mild ischemia was detected after the operation in $14.3 \%$ of the patients either by stress electrocardiography or by thallium 201 scintigraphy with exercise or dipyridamole loading. Clinical angina persisted or recurred in $6.5 \%$ of the patients. Three of these 11 patients underwent a second coronary bypass operation (one in the ITA group and two in the SVG group), and another patient in the SVG group had percutaneous transluminal coronary angioplasty to the graft. Myocardial infarction occurred in $2.4 \%$, ventricular arrhythmias in $1.2 \%$, and other cardiac events (pericardial hematoma and late mitral valve replacement) in $1.2 \%$. The cardiac outcome was unreported in $3.0 \%$. Postoperative ischemic events were relatively prevalent ( $24.4 \%$ ) in this series, but no difference in the prevalence of nonfatal cardiac events (angina, ischemia, myocardial infarction, and arrhythmias) was noted between the ITA group (24.6\%) and the SVG group (24.1\%).

\section{Discussion}

Kawasaki disease, or mucocutaneous lymph node syndrome, was first reported in Japan by Kawasaki and associates ${ }^{15}$ as a new pediatric disease in 1967 and is currently recognized worldwide. More than 100,000 patients with this disease have been documented in Japan.
Although the etiology of Kawasaki disease remains unknown, the development of postinflammatory coronary aneurysms and subsequent coronary artery obstruction have been proved to cause ischemic heart disease in children. Fortunately, pediatric ischemic heart disease is not prevalent ( $2 \%$ to $3 \%$ of patients with Kawasaki disease), but once myocardial infarction develops in children with severe coronary lesions, the prognosis is worse than was previously realized. According to Kato, Ichinose, and Kawasaki, ${ }^{16}$ the mortality rate in children with myocardial infarction induced by Kawasaki disease $(n=195)$ is $22 \%$ after the first infarct, $66 \%$ after the second infarct, and $87 \%$ after the third infarct. Accordingly, the development of surgical treatment for this disease was instrumental in preventing premature death and improving the quality of life of the afflicted children. ${ }^{17}$ The standard indications for surgical treatment have been reported elsewhere. ${ }^{18}$

As illustrated in Fig. 2, obstruction generally occurs in the proximal portions of the major coronary arteries. This fact increases the possibility of successful surgical treatment of the sequelae of coronary inflammation resulting from Kawasaki disease. Moreover, it is of interest that the incidence of lesions in each part of the coronary system closely resembles that for atherosclerotic coronary artery disease in adults.

During the development of surgical treatment for postinflammatory coronary lesions, it was soon realized that the patency of autologous SVGs was unsatisfactory in children. ${ }^{3-5}$ This was confirmed by the present study, 
which included a larger number of grafts. SVG patency fell sharply to $46.2 \% \pm 6.3 \%$ during the first few years after surgery. Because the SVG was first used for myocardial revascularization for Kawasaki disease, ${ }^{1-5}$ followed by the relatively recent use of arterial conduits, $5,8,10-14$ the total follow-up is shorter in the latter group. This discrepancy may influence the validity of comparing the two surgical approaches with different conduits. However, actuarial comparative analysis of the data used in the present study can eliminate, to some extent at least, the shortcomings of a comparison between two groups with different follow-up periods. Graft patency differs not only according to the graft material, but also according to the target vessel and the severity of the coronary obstruction. In the present analysis, no difference was identified in the severity of obstruction of the target vessel for arterial or venous grafts. However, venous grafts were used approximately 2.5 times more frequently than arterial grafts for the RCA and left circumflex system (Table IV), because the arterial graft was used exclusively for the LAD in all patients in the ITA group. The arterial graft showed a significantly higher patency rate than venous grafts for the RCA and left circumflex, as well as the coronary arteries as a whole including the LAD (Figs. 4 and 5). Also, the fact that the early patency rate for venous grafts 1 month after operation was comparable with that for arterial grafts ${ }^{19}$ may explain that differences in target vessels and the severity of obstruction have less of an effect on long-term patency than differences in graft materials.

It is noteworthy that the SVG patency rate in patients under 7 years of age was very low at $27.7 \% \pm 8.1 \%$ (Fig. 6). By contrast, the rate in patients over 8 years of age was significantly higher at $65.4 \% \pm 7.9 \%(p<0.01)$ (Fig. 7). Although the reasons for the poor patency of SVGs in small children remain unclear, several factors can be suggested. First, intimal hyperplasia of the SVG may occur faster in rapidly growing children with high serum levels of growth hormone. Second, technical precision becomes more important, particularly with regard to aortic anastomoses, in small children. Third, the isolated SVG is unable to grow along with the growth of the child. ${ }^{19}$ Degenerative changes of the heterologous valve implants also occur earlier in children than in adults, suggesting that some metabolic mechanisms specific to children may be responsible for the high attrition rate of autologous but isolated SVGs. Poor patency of SVGs was also reported by El-Said and coworkers ${ }^{20}$ in children with nonatheromatous coronary anomalies. Thus poor SVG patency appears to be common to all children, not only in children with Kawasaki disease.

In a search for better grafts, the ITA was tested in 1983 in a 6-year-old boy. ${ }^{10}$ Because good patency of ITA grafts and an excellent potential for their linear and circumferential enlargement in accordance with the growth of the child were reported, ${ }^{19}$ this graft has been used with increasing frequency in Japan., 12-14, 21 Bilateral use of the ITA also appears to be safe and can be recommended whenever indicated, because it does not adversely affect development of the chest wall in children. ${ }^{21}$ The GEA has also been used recently with favorable results. ${ }^{11-13}$ In the present cooperative study, the long-term patency of ITA and GEA grafts was significantly superior to that of SVGs, and this was particularly true in children less than 7 years of age. The arterial graft patency rate was approximately $77 \%$ in this study, which was somewhat lower than the rate in the series of the first author (S.K.), where the actuarial ITA patency rate $(n=65)$ was $89.3 \% \pm 5.7 \%$ and the SVG patency rate $(n=12)$ was $32.6 \% \pm 17.3 \%$ at 85 months $(p=$ not significant versus mean follow-up time in the cooperative study) in 38 consecutive patients with a mean age of $9.2 \pm 3.9$ years $(1$ to 19 years) at operation ( $p=$ not significant versus mean age in the cooperative study). Thus there is no doubt that arterial grafts, particularly the ITA, have a much better long-term patency than SVGs in children.

Poor long-term patency of SVGs was reflected by a high late cardiac death rate of $13.0 \%$, and the absence of an ITA graft to the LAD was a significant $(p<0.003)$ factor predicting late death in the present study. A younger age at operation was also a significant $(p<0.05)$ factor predicting early and late death. This fact may be related to the technical difficulty of the operation as well as the high attrition rate of SVGs in smaller children, resulting in incomplete myocardial revascularization and subsequent cardiac death. The number of grafts per patient was only $1.7 \pm 0.7$ in the two groups and the number of vessels involved was 2.0 to 2.2 per patient. These facts also demonstrate that incomplete revascularization was common, mostly because of the technical difficulty of operating on the small vessels of young children. This and the high graft occlusion rate can explain the prevalence of postoperative cardiac events $(24.4 \%)$ as shown in Fig. 8, although associated myocarditis may play a significant role in the increased frequency of abnormal electrocardiograms or abnormal thallium 201 uptake. $22-24$

In the present study, the ITA and probably the GEA have been demonstrated to be the grafts of choice for myocardial revascularization in children. In our minds, the use of vein grafts for pediatric myocardial revascularization should be avoided if at all possible. These results can probably also be applied to pediatric bypass grafting for nonatherogenic coronary artery disease or anomalies 
other than Kawasaki disease. ${ }^{25-27}$ Examination of left ventricular function during exercise reveals postoperative improvement in many children, ${ }^{28}$ and $70 \%$ of the children are able to participate in the full school sports program. ${ }^{17}$ Because most of these children were prohibited to exercise at school before the operation, we conclude that bypass grafting is effective in improving the quality of life of children with severe coronary sequelae of Kawasaki disease and can reduce the likelihood of cardiac death, particularly when the major coronary artery is well revascularized by a graft with a long duration of patency like an ITA graft. Further detailed analysis by the multivariate logistic analysis and a longer period of follow-up are needed. Ideally, follow-up should continue into and beyond the age when atherosclerotic coronary artery disease supervenes.

We gratefully appreciate and acknowledge all the surgeons and pediatric cardiologists in this multicenter cooperative study under the auspices of the Japanese Ministry of Health and Welfare for their contributions in providing patient data.

\section{REFERENCES}

1. Kitamura S, Kawashima Y, Miyamoto K, et al. Multiple coronary aneurysms resulting in myocardial infarction in a young man. J THORAC CARDIOVASC SURG 1975;70:290-7.

2. Kitamura S, Kawashima Y, Fujita T, et al. Aortocoronary bypass grafting in a child with coronary artery obstruction due to mucocutaneous lymph node syndrome. Circulation 1976;53:1035-40.

3. Kitamura S, Kawachi K, Harima R, Sakakibara T, Hirose H, Kawashima Y. Surgery for coronary heart disease due to mucocutaneous lymph node syndrome (Kawasaki disease). Am J Cardiol 1983;51:444-8.

4. Suma K, Takeuchi Y, Shiroma K, et al. Early and late postoperative studies in coronary arterial lesions resulting from Kawasaki's disease in children. J THORAC CARDIOVASC SURG 1982;84:2249.

5. Suzuki A, Kamiya T, Ono Y, Okuno M, Yagihara T. Aortocoronary bypass surgery for coronary arterial lesions resulting from Kawasaki disease. J Pediatr 1990;1 16:56773.

6. Sandiford FM, Vargo TA, Shih JY, Pelargonio S, McNamara DG. Successful triple coronary artery bypass in a child with multiple coronary aneurysms due to Kawasaki's disease. J Thorac Cardiovasc Surg 1980;79:283-7.

7. Mains C, Wiggins J, Groves B, Clarke D. Successful therapy for a complication of Kawasaki's disease. Ann Thorac Surg 1983;35:197-200.

8. Myers JL, Gleason MM, Cyran SE, Baylen BG. Surgical management of coronary insufficiency in a child with Kawasaki's disease: use of bilateral internal mammary arteries. Ann Thorac Surg 1988;46:459-61.

9. D'Amico TA, Sabiston DC Jr. Kawasaki's disease. In:
Sabiston DC Jr, Spencer FC, eds. Surgery of the chest. Philadelphia: WB Saunders, 1990:1759-66.

10. Kitamura S, Kawachi K, Oyama C, et al. Severe Kawasaki heart disease treated with an internal mammary artery graft in pediatric patients: a first successful report. J THORAC CARDIOVASC SURG 1985;89:860-6.

11. Takeuchi Y, Gomi A, Okamura Y, Mori H, Nagashima M. Coronary revascularization in a child with Kawasaki disease: use of right gastroepiloic artery. Ann Thorac Surg 1990;50:294-6.

12. Kosuga $K$, Isomura $T$, Hisatomi $K$, et al. Revascularization in Kawasaki disease using internal thoracic and gastroepiploic arteries. In: Fournial G, Glock Y, Roux D, Dalous P, eds. Internal thoracic artery for myocardial revascularization. Toulouse, France: Imprimeries Fournie, 1990:297304.

13. Kitamura S, Kawachi K, Seki T, Morita R. Use of internal thoracic artery graft in congenital or acquired pediatric coronary artery diseases. In: Fournial G, Glock Y, Roux D, Dalous $\mathrm{P}$, eds. Internal thoracic artery for myocardial revascularization. Toulouse, France: Imprimeries Fournie, 1990:223-40.

14. Isomura $T$, Hisatomi $K$, Hirano $A$, Ohishi $K$, Inoue $O$, $\mathrm{Kato} \mathrm{H}$. The internal thoracic artery and its branches after coronary artery anastomoses in pediatric patients. J Cardiac Surg 1992;7:225-30.

15. Kawasaki T, Kosaki I, Okawa S, Shigematsu I, Yanagawa H. A new infantile acute febrile mucocutaneous lymph node syndrome (MLNS) prevailing in Japan. Pediatrics 1974;54:271-6.

16. Kato H, Ichinose E, Kawasaki T. Myocardial infarction in Kawasaki disease: clinical analyses of 195 cases. J Pediatr 1986;108:923-7.

17. Kitamura S. Surgical management for cardiovascular lesions in Kawasaki disease. Cardiol Young 1991;1:240-53.

18. Kato H, Kitamura S, Kawasaki T. Guidelines for treatment and management of cardiovascular sequelae in Kawasaki disease. Heart Vessels 1987;3:50-4.

19. Kitamura S, Seki T, Kawachi K, et al. Excellent patency and growth potential of internal mammary artery grafts in pediatric coronary bypass surgery: new evidence for a "live" conduit. Circulation 1989;78(Suppl):129-39.

20. El-Said GM, Ruzyllo W, William RL, et al. Early and late result of saphenous vein graft for anomalous origin of left coronary artery from pulmonary artery. Circulation 1973; 48(Suppl):III2-6.

21. Kitamura S, Kawachi K, Seki T, et al. Bilateral internal mammary artery grafts for coronary bypass operations in children. J THORAC CARDIOVASC SURG 1990;99:708-15.

22. Kitamura S, Kawashima Y, Kawachi K, Kozuka T, Fujita $T$, Manabe $H$. Left ventricular function in patients with coronary arteritis due to acute febrile mucocutaneous lymph node syndrome or related diseases. Am J Cardiol 1977;40:156-64.

23. Newburger JW, Sanders SP, Burns JC, Parness IA, Beiser AS, Colan SD. Left ventricular contractility and function 
in Kawasaki syndrome: effect of intravenous gamma globulin. Circulation 1989;79:1237-46.

24. Takahashi M. Myocarditis in Kawasaki syndrome: A minor villain? Circulation 1989;79:1398-400.

25. Cohen AJ, Grishkin BA, Helsel RA, Head HD. Surgical therapy in the management of coronary anomalies: emphasis on utility of internal mammary artery grafts. Ann Thorac Surg 1989;47:630-7.

26. Fortune RL, Baron PJ, Fitzgerald JW. Atresia of the left main coronary artery: repair with left internal mammary bypass. J ThORAC CARDIOvasc Surg 1987;94:150-1.

27. Kitamura $S$, Kawachi K, Nishii $T$, et al. Internal thoracic artery grafting for congenital coronary malformations. Ann Thorac Surg 1992;53:513-6.

28. Kawachi K, Kitamura S, Seki T, et al. Hemodynamics and coronary blood flow during exercise after coronary artery bypass grafting with internal mammary arteries in children with Kawasaki disease. Circulation 1991;84:618-24.

\section{Discussion}

Dr. Hillel Laks (Los Angeles, Calif.). What are your thoughts about the causes for the higher occlusion rate for the SVGs in children as compared with adults? One would assume that in children atherosclerosis is not a major factor. What do you think are the other causes for graft occlusion in the SVG group?

Dr. Kitamura. Let me answer that question, Dr. Laks, through my personal experience with nearly 40 patients. SVG patency is equal to arterial graft patency at 1 month after operation. However, the attrition rate of SVGs is quite fast and high compared with that of the arterial grafts. This phenomenon has been demonstrated in adult patients with atherosclerotic coronary disease, but the pediatric patients without atherosclerotic disease have a more intensified or exaggerated type of attrition of SVGs. I do not know why. Maybe in growing children intimal hyperplasia of isolated SVGs is much faster than in adults. Also, some degenerative changes of isolated SVGs may be more prevalent, just as a xenograft valve degenerates much faster in children than in adults. I do not know the real reason, but intimal thickening occurs much more quickly in growing children than in adults.

Dr. Serafin Y. DeLeon (Maywood, Ill.). My dilemma with Kawasaki disease is when to operate. There is not much question in patients who already have ischemic changes or myocardial infarction. However, when do you intervene in patients who are free of symptoms but have horrible-looking coronary arteries on echocardiography or angiography?

Dr. Kitamura. As Dr. DeLeon said, we cannot get the clinical signs well from the child. The child usually complains just of upper abdominal pain at the time of myocardial infarction, and that is not reliable. We rely on the echocardiographic study followed by an angiographic study. We do not operate on the aneurysm alone, but we do operate on the patient with tight stenosis at the proximal site of a major coronary artery with evidence of ischemia or myocardial infarction. Ischemia can be detected by a thallium study with dipyridamole loading in children. We need a positive ischemic sign plus angiographic evidence of tight stenosis of a major proximal coronary artery. We take these two signs as the most important indications for surgery, because patients die suddenly at school, and autopsy shows evidence similar to that demonstrated by angiography before death.

Dr. Ronald C. Elkins (Oklahoma City, Okla.). First, how accurate do you believe thallium stress tests are in this age group for diagnosing ischemia? Second, what is your choice of bypass graft for the patient who has a lesion of the distal RCA or of the posterior descending coronary artery.

Dr. Kitamura. To answer Dr. Elkins' last question first, I now prefer using the GEA to the distal RCA, particularly the posterior descending area. The right ITA can reach the crux area of the RCA most of the time. I have been trying to use a right ITA whenever it can reach the point of anastomosis required; otherwise I use the GEA for that purpose even in small children. I successfully used a GEA for the posterior descending artery in a 1-year-old boy.

If the patient is older than 6 or 7 years old, we usually subject the patient to a treadmill test. In patients younger than that age, we judge the ischemic change by giving dipyridamole and thallium. That may be the only way we can judge ischemic change. We have not done any positron emission tomographic study or any other more advanced and sophisticated study. They are not available often in Japan.

Dr. Hafil Abdulgan. (Jakarta, Indonesia). I have two questions. First, is there any difference in the results obtained with interrupted or continuous suture? Second, is there any special way of administering myocardial protection in your series-that is, do you use retrograde cardioplegia?

Dr. Kitamura. Yes, Dr. Abdulgani, I use two 8-0 Prolene monofilament sutures (Ethicon, Inc., Somerville, N.J.) to anastomose arterial grafts to the coronary artery. Usually I use running over-and-over sutures, not interrupted sutures. In my mind, it does not make any difference. I have already reported the growth of an anastomotic site of the graft by using this technique (J ThoraC CardiovasC Surg 1990;99:708-15). I know that many surgeons try to put interrupted sutures on one side of the anastomosis, but I do not think it is necessary for the anastomotic site to grow if it is stitched carefully and meticulously.

In response to the second question regarding myocardial protection during operation, I use the same method for children that I use for adults. I use antegrade blood cardioplegia, and recently I have started using retrograde cardioplegia in association with antegrade cardioplegic infusion. I use blood cardioplegia most of the time and moderate hypothermic cardiopulmonary bypass.

Dr. John E. Mayer (Boston, Mass.). What is the interval in these patients between the acute, febrile, rash phase of this illness and the time of the operation? Have you ever operated in the relatively acute phase in which the aneurysms are large and frequently filled with thrombus, which is at risk for embolizing into the distal coronary circulation?

Dr. Kitamura. The interval between the acute illness and operation varies significantly from 1 year to 22 years. As I said, the indication for operation is coronary stenosis or obstruction, not the aneurysm itself, and the stenosis occurs at the inflow or outflow portion of the aneurysm in the majority of the patients over the years. It takes at least 1 year, and in my series the shortest period between acute illness and operation was 1 year. The structure of the aneurysm changes remarkably within a year. We see the big aneurysm first, but it changes in shape and size, usually getting smaller, and sometimes it becomes obstructed. For this reason, I would recommend waiting with care for 
at least 1 year before operating unless ischemic events require urgent surgery soon after the acute illness, but I have not experienced this scenario.

\section{Appendix}

Department of Surgery, Department of Cardiovascular Surgery or Department of Pediatrics of the forty Participating Institutions in 1991: Aomori Central Hospital, Anjo Kosei Hospital, Akita University Hospital, Osaka Medical College Hospital, Osaka University Hospital, Osaka Prefectural Hospital, Kanazawa University Hospital, Kawasaki City Hospital, Kansai Medical College Hospital, Kansai Rosai Hospital, Kitazato University Hospital, Kyoto University Hospital, Kinki University Hospital, Kyushu University Hospital,
Kurume University Hospital, Kobe Central Hospital, Matsudo City Hospital, National Saitama Hospital, Saiseikai Utsunomiya Hospital, Sakakibara Memorial Hospital, Mitsui Memorial Hospital, Hiroshima City Hospital, Showa University Hospital, St. Marianna University Hospital, Seirei Hamamatsu Hospital, Chiba Tsurumai Center Hospital, Tokyo Women's Medical College Second Hospital, Toyama Medical and Pharmaceutical University Hospital, Toranomon Hospital, Nihon University Hospital, Mie University Hospital, Hamamatsu Medical College Hospital, Himeji Cardiovascular Center Hospital, Fukui Cardiovascular Center Hospital, Fukuoka University Hospital, Yokohama City University Hospital, Kanto Teishin Hospital, Heart Institute of Japan Hospital of the Tokyo Women's Medical College, National Cardiovascular Center Hospital, and Nara Medical College Hospital

\section{Bound volumes available to subscribers}

Bound volumes of THE JOURNAL OF THORACIC AND CARDIOVASCULAR SURGERY are available to subscribers (only) for the 1994 issues from the Publisher, at a cost of $\$ 82.50$ for domestic, $\$ 106.28$ for Canadian, and $\$ 100.50$ for international subscribers for Vol. 107 (January-June) and Vol. 108 (July-December). Shipping charges are included. Each bound volume contains a subject and author index and all advertising is removed. Copies are shipped within 60 days after publication of the last issue of the volume. The binding is durable buckram with the JOURNAL name, volume number, and year stamped in gold on the spine. Payment must accompany all orders. Contact Mosby-Year Book, Inc., Subscription Services, 11830 Westline Industrial Drive, St. Louis, Missouri 63146-3318, USA; phone 1 (800) 4534351 or $(314) 453-4351$.

Subscriptions must be in force to qualify. Bound volumes are not available in place of a regular JourNaL subscription. 